\title{
Departure Queue Prediction for Strategic and Tactical Surface Scheduler Integration
}

\author{
Shannon Zelinski and Robert Windhorst \\ Aerospace High Density Operations Branch \\ NASA Ames Research Center \\ Moffett Field, CA, 94035 \\ Shannon.J.Zelinski@nasa.gov, Robert.D.Windhorst@,nasa.gov
}

\begin{abstract}
A departure metering concept to be demonstrated at Charlotte Douglas International Airport (CLT) will integrate strategic and tactical surface scheduling components to enable the respective collaborative decision making and improved efficiency benefits these two methods of scheduling provide. This study analyzes the effect of tactical scheduling on strategic scheduler predictability. Strategic queue predictions and target gate pushback times to achieve a desired queue length are compared between fast time simulations of CLT surface operations with and without tactical scheduling. The use of variable departure rates as a strategic scheduler input was shown to substantially improve queue predictions over static departure rates. With target queue length calibration, the strategic scheduler can be tuned to produce average delays within one minute of the tactical scheduler. However, root mean square differences between strategic and tactical delays were between 12 and 15 minutes due to the different methods the strategic and tactical schedulers use to predict takeoff times and generate gate pushback clearances. This demonstrates how difficult it is for the strategic scheduler to predict tactical scheduler assigned gate delays on an individual flight basis as the tactical scheduler adjusts departure sequence to accommodate arrival interactions. Strategic/tactical scheduler compatibility may be improved by providing more arrival information to the strategic scheduler and stabilizing tactical scheduler changes to runway sequence in response to arrivals.
\end{abstract}

Keywords-Departure metering, surface scheduling, departure queue prediction.

\section{INTRODUCTION}

NASA is committed to demonstrating an Integrated Arrival, Departure, Surface (IADS) concept at Charlotte Douglas International Airport (CLT) within the next few years [1]. The surface scheduling capability of the proposed IADS system includes a strategic component to enable planning and collaborative decision making among airlines and air traffic managers, and a tactical component to enable flexibility and maximize efficiency and throughput. The strategic scheduler derives from the Surface-Collaborative Decision Making (SCDM) concept's Departure Reservoir Management (DRM) capability [2]. The tactical scheduler derives from a first-comefirst-served (FCFS) with heuristics version of NASA's Spot And Runway Departure Advisor (SARDA) [3,4]. Thus far the strategic and tactical scheduling components have been developed separately and research is required to study how they will interact. The end goal of these schedulers is the same, to maximize both the efficiency and predictability of surface operations under various operational constraints. However, they use different metrics to drive their scheduling solutions to this shared goal. Whereas the tactical scheduler uses flight delay to drive its schedule, the strategic scheduler uses queue length. Here queue length can be defined as the number of aircraft using or waiting to use surface resources. As a first step toward integrating the tactical and strategic surface schedulers, this paper explores the effect of tactical scheduling on strategic scheduling.

Both schedulers attempt to meter surface traffic by assigning target release times. A Target Off Block Time (TOBT) is the suggested time to release a departure for gate pushback. A Target Movement Area entry Time (TMAT) is the suggested time to release a departure from the spot and transition from the airline controlled ramp area of the surface to air traffic controlled taxiways. The tactical scheduler assigns TOBTs no more than 15 minutes in advance. TOBTs are updated every 10 seconds until they are within 2 minutes of current time, when they are frozen. When the strategic scheduler predicts runway queues will exceed an upper threshold, it assigns TMATs as much as 90 minutes in advance as part of a Departure Metering Program (DMP). An implemented DMP may be extended, or adjusted if predictions change. However, unlike tactical TOBTs, strategic individual TMATs are not as flexible to change in response to minor uncertainty. The envisioned integration of these schedulers expects that the strategic TMATs will be given to the tactical scheduler, which then assigns TOBTs designed to meet the strategic TMATs within a defined compliance time range. If successful, the strategic adds an element of stability and predictability to surface metering, enabling a collaborative decision making mechanism by which air carriers can negotiate on scheduling decisions (e.g. slot swapping) for equitability. Meanwhile, the tactical scheduler is still allowed to make small adjustments to maximize efficiency in response to uncertainty (e.g. pushback readiness, taxi-time, arrival operations). An important research question is how large the compliance time range surrounding strategic TMATs should be to allow tactical improvements. The answer to this question will be influenced not only by uncertainties in surface trajectory predictions, but by the inherent differences in how the strategic and tactical surface schedulers generate their respective metering times.

This paper analyzes the effect of tactical scheduling on strategic scheduler predictability. Section II describes the 
simulation environment and strategic and tactical schedulers in detail. In section III, strategic queue predictions and target times are analyzed using three different queue definitions to drive the strategic scheduler. Conclusions are presented in section IV.

\section{Simulation}

\section{A. Surface Operations}

The Surface Operations Simulator and Scheduler (SOSS) [5] is used to simulate surface operations at CLT both with and without tactical surface metering. In the tactical surface metering simulations, SOSS holds aircraft at the gate until their target gate departure times. Without surface metering, SOSS releases aircraft from the gate as soon as they are ready. SOSS simulates both departure and arrival aircraft movements within a network of nodes and links representing gates, ramp, taxiways, and runways. Aircraft are allowed to progress along predefined routes through the node-link network as long as they do not violate separations constraints specific to each part of the network. Fig. 1 shows a map view of the SOSS CLT surface model, highlighting characteristics of South flow operations. Runways are labeled in light blue and yellow to indicate their predominant use for arrivals and departures respectively. When departure demand exceeds runway capacity, departure queues form along the taxiways to the right of $18 \mathrm{C}$ and to the left of $18 \mathrm{~L}$, indicated by green arrows. Crossing light blue and yellow arrows highlight runway constraints where arrivals impact departure operations and consequently departure queue length. Intersecting runways $18 \mathrm{~L}$ and 23 cause arrivals on 23 to impact departures on $18 \mathrm{~L}$. Converging runways $18 \mathrm{C}$ and 23 cause arrivals on 23 to impact departures on $18 \mathrm{C}$. Arrivals on $18 \mathrm{R}$ must cross $18 \mathrm{C}$ as they taxi to the gates, impacting departures on $18 \mathrm{C}$.

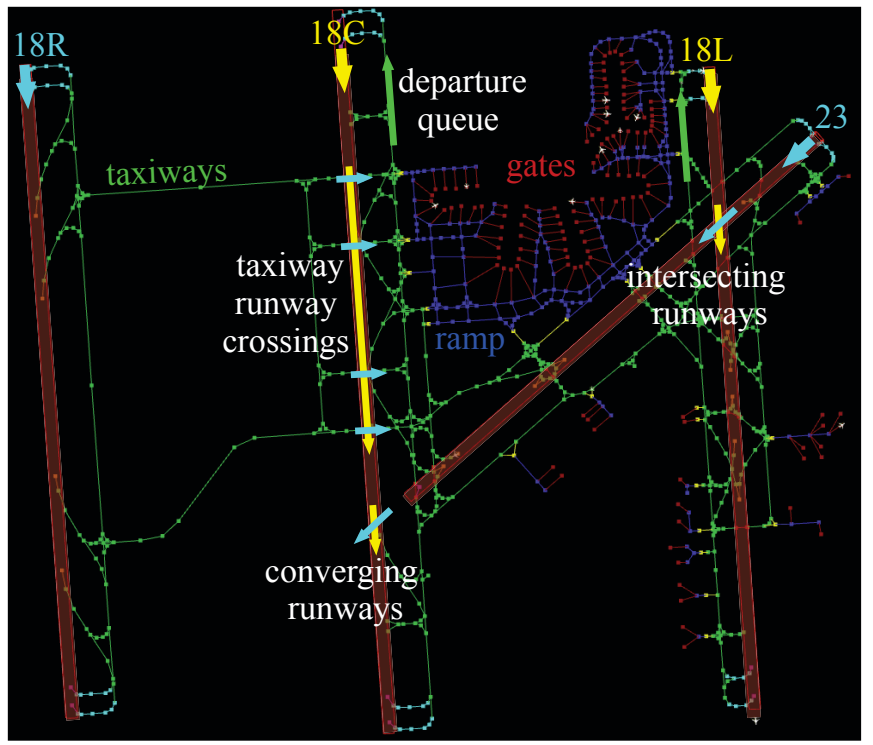

Fig. 1. CLT surface model.

Although SOSS has the capability of modeling uncertainty in various areas of the airport surface, uncertainty was not modeled for the purposes of this analysis.

\section{B. Traffic Scenario}

A SOSS traffic scenario is a list of departure and arrival flight information including aircraft type, flight plan, runway and gate assignment, and time the flight enters the simulation. Arrival and departure flight plans include the Standard Terminal Arrival Route (STAR) or Standard Instrument Departure (SID) respectively. Arrivals enter the simulation at the final approach fix and departures enter the simulation at the gate at their published pushback time or p-time. A four-hour scenario was developed based on CLT surface surveillance data from March 11, 2016. This was a relatively high traffic volume day with low weather impact when CLT was operating in South flow most of the day. Surface surveillance data was used assign the actual gate and runway used by each flight. Nominal routings between gates and runways within the SOSS node-link network were generated using rules learned from CLT ramp controllers.

Fig. 2 shows the demand for each runway as numbers of arrivals and departures per 15-minutes. As a central hub for American Airlines, CLT traffic is characterized by cycles of a departure push (departure demand peak) vacating the gates, followed closely by an arrival rush (arrival demand peak) occupying the gates, followed by a lull in operations when passengers transit between connecting flights. The four-hour scenario in Fig. 2 covers two such cycles. Analysis of strategic scheduler calculations with up to 90-minutes lookahead cannot begin until at least 90 minutes into the simulation. This fourhour scenario allows analysis of a complete departure-arrival cycle beginning at 120 minutes.

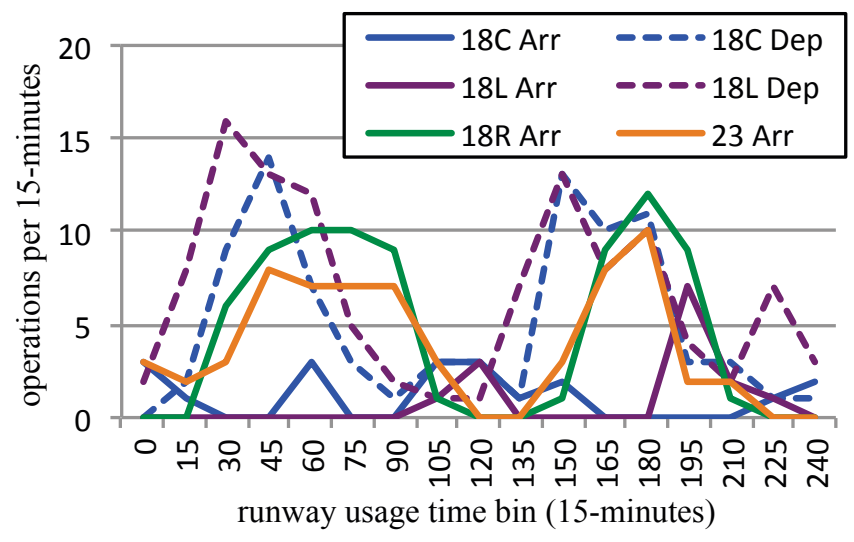

Fig. 2. Runway demand for the four-hour scenario.

\section{Strategic Surface Scheduler}

Table I summarizes the abbreviations used for times at surface points throughout this section.

TABLE I. ABbreViations For Times AT Surface POINTS

\begin{tabular}{|l|l|l|l|}
\hline \multicolumn{2}{|c|}{ Abbreviation Prefix $=$ Time Type } & \multicolumn{2}{c|}{ Abbreviation Suffix $=$ Surface Point } \\
\hline A & Actual & OBT & Off Block Time \\
\hline E & Earliest & MAT & Movement Area entry Time \\
\hline P & Predicted & QET & Queue Entry Time \\
\hline T & Target & TOT & Take Off Time \\
\hline
\end{tabular}


The strategic surface scheduler is modeled after the DRM, which uses a manually input target queue length per runway to calculate TMATs. The DRM periodically calculates and compares queue predictions to the target queue length, and recommends a DMP including TMATs for all flights within the DMP active time period, only when the queue is predicted to exceed the target queue length by a user defined amount.

The strategic scheduler for this analysis differs from the DRM in that TOBTs are calculated rather than TMATs so that they may be compared with tactical scheduler generated TOBTs. The strategic TOBT esimate is simply TMAT minus unimpeded ramp transit time. Both strategic and tactical TOBT calculations begin by generating Predicted Take Off Times (PTOTs) and back calculating the target times to achieve the desired queue length. However, the DRM employs a more complex method of assigning PTOT slots FCFS by Initial Off Block Time to inhibit gaming as airlines update their Earliest Off Block Time (EOBT) estimates. For the purposes of this analysis, the calculation of PTOTs is made simpler by assuming EOBTs are not updated by the airlines. In this analysis, DMPs are not implemented. Therefore, the strategic scheduler continually updates both queue predictions and TOBTs every minute.

\section{1) Queue Prediction}

The strategic scheduler calculates a range of queue predictions from current time to 90 -minute lookahead in oneminute intervals. These queue predictions are updated every minute using new current state information.

Three different definitions of queue length for a given runway are considered. Queue $\mathrm{q} 1$ is the number of active aircraft on the ramp and taxiways. Queue q2 is the number of aircraft on just the taxiways. Queue $\mathrm{q} 3$ is the number of aircraft on the taxiways waiting in line to use the runway. Note that the flights in $\mathrm{q} 3$ are a subset of $\mathrm{q} 2$, which are a subset of q1.

For all queue definitions, an aircraft exits the queue at its Actual Take Off Time (ATOT). Actual Queue Entry Times (AQETs) for each queue definition are as follows. A flight's AQET1 is when it is released from departure gate, also know as the Actual Off Block Time (AOBT). A flight's AQET2 is when it is released from the spot and transitions from the ramp to the active movement area (taxiways), also known as the Actual Movement Area entry Time (AMAT). A flight's AQET3 is when it has exceeded its unimpeded taxi out time since entering the active movement area. This is equal to the flight's last updated Earliest Take Off Time (ETOT) before AMAT.

The queue prediction method begins by calculating Earliest Queue Entry Times (EQETs) and Predicted Take Off Times (PTOTs) for each flight. For all flights currently at the gate: EQET1 equals the EOBT, which is the maximum of the flight's p-time and the current time; EQET2 equals the Earliest Movement Area entry Time (EMAT) projected from EOBT; and EQET3 equals the ETOT projected from EMAT. For all flights currently on the ramp, AQET1 is used instead of EQET1 and EQET2 is the updated EMAT projected from current position. For all flights currently on the taxiways, AQET2 is used instead of EQET2. EQET1 and EQET2 are updated every minute as EOBT and EMAT are updated.
Whereas EQET3 is updated when the flight is still at the gate or in the ramp, it is not updated once the flight enters the active movement area, and this last EQET3 will in fact become AQET3 when current time catches up to it. Fig. 3 shows how EQETs update until they become AQETs as the flight transitions from gate to ramp to taxi. Notice how when the flight is in taxi, ETOT continues to update, whereas AQET2 remains frozen from the time the flight entered the active movement area (AMAT).

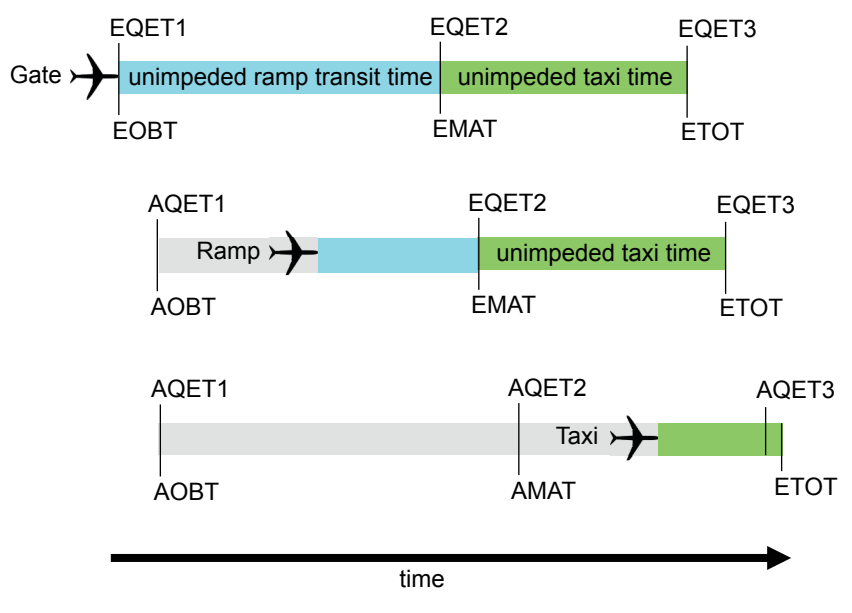

Fig. 3. Queue entry time updates.

Flight PTOTs are calculated by scheduling flights to the runway in FCFS fashion, taking into account departure runway spacing constraints. The FCFS sequence is determined by first sorting all flights by ETOT and modifying to ensure that precedence constraints are met. For example, a fast aircraft lined up behind a slow aircraft on the same taxiway may have an earlier ETOT, but the slow aircraft has precedence and must be scheduled first because it is physically impossible for the fast aircraft behind it to pass on the taxiway.

Departure runway spacing constraints include wake vortex separation, departure fix miles-in-trail, and runway departure rate. The strategic scheduler does not consider arrival specific spacing constraints (imposed by intersecting or converging arrival runways, or arrivals landing on or crossing the scheduled runway) because accurate arrival runway assignments and landing times are not expected to be available to the strategic surface scheduler two hours in advance. Arrivals are instead accounted for with runway departure rate spacing constraints. Departure rate is envisioned to be a user defined input to the strategic scheduler manually updated by the Departure Reservoir Coordinator as airport configuration and other surface conditions change. When arrivals are expected to impact departure operations on a particular runway, a lower departure rate would be entered, which would increase the minimum inter-departure spacing constraint.

The current queue lengths per runway, are the numbers of flights that have an AQET but no ATOT (entered but not yet exited the queue). Predicted queue lengths per runway are calculated by sorting all EQETs and PTOTs by increasing time. Then for each EQET and PTOT, the current queue length is incremented or decremented respectively, and set for the 
associated EQET or PTOT. The predicted queue length is then retrieved from the list of EQETs and PTOTs and recorded every 60 seconds out to 90 minutes from current time.

\section{2) Target Off Block Time Calculation}

Strategic TOBTs are back calculated from Target Queue Entry Times (TQETs) designed to maintain the target queue length. For a target queue length $\mathrm{Q}$, the TQET of the $i$ th flight in runway takeoff sequence is equal to the PTOT of the ( $i$-Q)th flight in sequence. If the ( $i$-Q)th flight has already exited the queue (taken off), then the TQET of the $i$ th flight equals its EQET. Unimpeded transit time is then subtracted from TQET to get TOBT, depending on the queue definition. TOBT1 is equal to TQET1. Unimpeded ramp transit time is subtracted from TQET2 to get TOBT2. Both unimpeded ramp transit and taxi times are subtracted from TQET3 to get TOBT3.

\section{Tactical Surface Scheduler}

The tactical surface scheduler is modeled after a FCFS version of SARDA. As with the strategic scheduler, TOBT calculation begins with assigning PTOTs to flights. Like the strategic scheduler, the tactical scheduler sequences flights for scheduling by sorting all flights by ETOT and modifying it to ensure that precedence constraints are met. Whereas the strategic scheduler considers only departures, the tactical scheduler also considers arrivals landing on intersecting and converging runways, as well as arrivals landing on or taxing across the scheduled runway. Arrival landing times are treated as hard constraints, but arrivals crossing the scheduled runway are sequenced along with the departures and scheduled in turn. In addition, the tactical scheduler attempts to resolve gate conflicts by prioritizing flights that would otherwise block the gate for incoming arrivals. As the tactical scheduler explicitly considers arrival specific runway spacing constraints, it does not use departure rate as a constraint.

After the tactical scheduler calculates PTOTs, it calculates a TOBT for each flight by subtracting unimpeded transit time (through ramp and taxi) and a delay buffer from its PTOT. The delay buffer varies linearly with the unimpeded transit time such that the buffer equals a constant $c$ multiplied by the unimpeded transit time. The constant $c$ is tuned to a particular airport configuration to provide just enough delay buffer to keep the runways from going dry. A delay buffer of 3\% was used for this study because this is was the buffer used in recent human-in-the-loop SARDA simulations of CLT [3].

The tactical scheduler calculates TOBTs for all flights with EOBT within 15 minutes of current time and updates every 10 seconds. When a flight's TOBT is within 2 minutes of current time, it is frozen and no longer updated.

\section{E. Strategic-Tactical Scheduler Interaction}

This study models the strategic scheduler in predictive mode, so there is no information passed from the strategic to the tactical scheduler. However, when the tactical scheduler assigns a TOBT to a flight, this tactical TOBT updates the EOBT the strategic scheduler uses to calculate queue predictions and strategic TOBTs. To facilitate analysis of open-loop strategic TOBTs with as little tactical information as possible, the strategic scheduler still calculates TOBTs for flights with tactical TOBTs. Final implementation of the strategic scheduler will likely directly use tactical TOBTs and other tactical scheduling information rather than recalculating them, but this is left for future study.

Fig. 4 diagrams how information is passed among SOSS and the tactical and strategic schedulers. Notice how information and parameters flow only into the strategic scheduler. Its outputs are metrics for analysis.

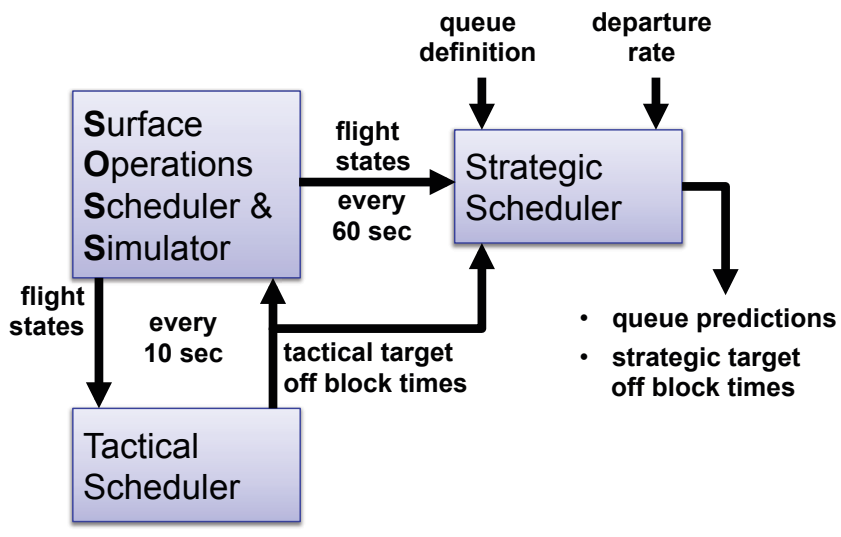

Fig. 4. Simulation information flow.

\section{RESULTS}

Several analyses were completed using the output of two SOSS simulations, one with and one without tactical surface metering, hereafter referred to as the "Tactical" and "Baseline" simulations, respectively. First the tactical scheduler performance results are presented, followed by strategic scheduler performance analyses of queue and TOBT prediction.

\section{A. Tactical Scheduler Performance}

Fig. 5 compares taxi times and delays between the baseline and tactical scheduling simulations. The boxes and whiskers show quartiles and means for all individual flight taxi times (transit time from gate to runway) and delays within each simulation.

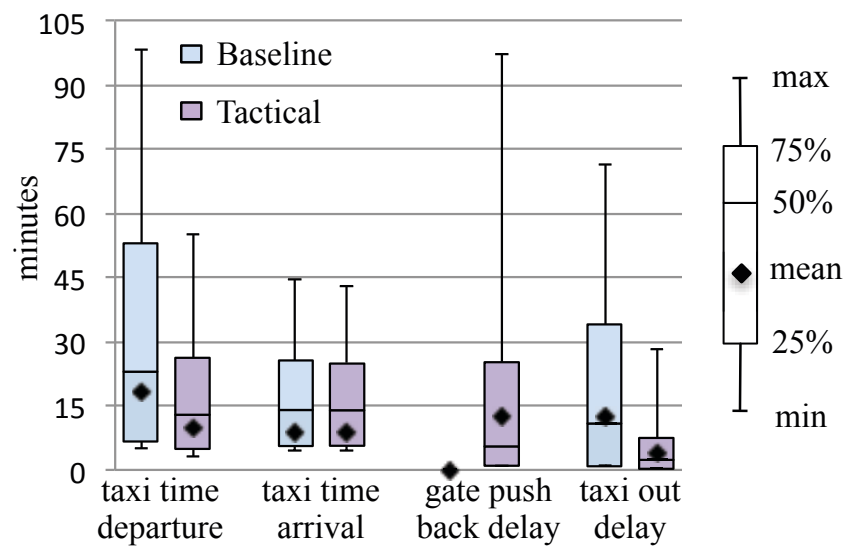

Fig. 5. Simulation taxi times and delays. 
The tactical scheduler substantially reduces departure taxi time without affecting arrival taxi time. It does this by moving most of the taxi delay to the gate where flights may wait with engines off, burning less fuel and producing less emissions. Not only is taxi delay reduced, but it is far more consistent, improving predictability and enabling the capability to coordinate in an IADS context.

Fig. 6 compares the queue lengths at runway $18 \mathrm{C}$ between simulations. Queue q1 is largest for both Baseline and Tactical simulations because this counts all flights in the ramp and taxiways, whereas q2 and q3 count only flights in the taxiways. Similarly, q2 is always greater than or equal to $\mathrm{q} 3$ as $\mathrm{q} 3$ is the subset of q2 flights that have exceed their unimpeded taxi time. The similarity between $\mathrm{q} 2$ and $\mathrm{q} 3$ indicates that most flights in the taxiways had exceeded their unimpeded taxi time. Compared to Baseline, the Tactical simulation substantially reduces all queue lengths by holding flights at the gate and delaying their queue entry times. However, Tactical simulation queue lengths are still quite erratic because the tactical scheduler does not target a desired queue length with it's method of departure metering.

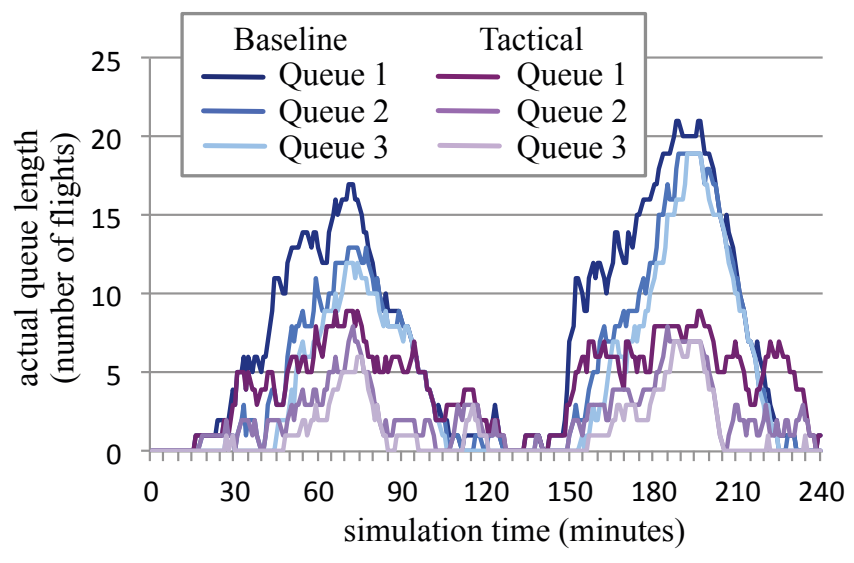

Fig. 6. Simulation queue lengths at $18 \mathrm{C}$.

Fig. 7 compares the departures per quarter hour at runway $18 \mathrm{C}$ between simulations.

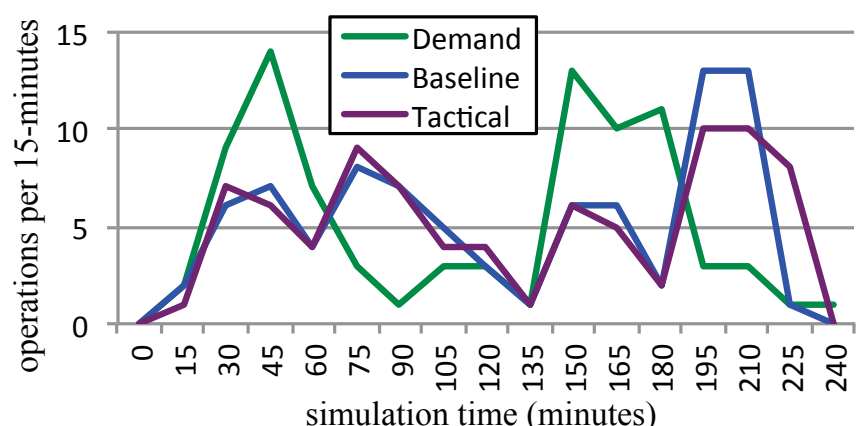

Fig. 7. Simulation demand and departure rates at $18 \mathrm{C}$.

Both simulations' departure rates are substantially lower than demand during the demand peaks, pushing more operations to later time bins as flights are delayed. The dips in departure rate at 60 and 180 minutes are due to arrival demand peaks interfering with departure operations. Most of the time, the tactical scheduler is able to produce a similar departure rate to that of the baseline except during the last hour when tactical scheduler departure rates are lower.

\section{B. Queue Prediction Analysis}

Departure rate is envisioned to be a user defined input to the strategic scheduler manually updated by the Departure Reservoir Coordinator as airport configuration and other surface conditions change. The departure rate is used as a flexible calibration factor to compensate for the lack of arrival runway usage rather than an explicit value. This analysis compares queue prediction accuracy between calibrated static and variable departure rate inputs.

Root mean square error was chosen to measure prediction accuracy. Let $e(i, j)$ be the queue prediction error for time $i$ at a lookahead time of $j$ minutes.

$$
e(i, j)=q(i, j)-q(i, 0)
$$

where $q(i, 0)$ is the actual queue length at time $i$ (i.e. the lookahead time is 0$)$ and $q(i, j)$ is the queue prediction for time $i$ at a lookahead time of $j$ minutes.

Let $\sigma\left(i,{ }^{*}\right)$ be the root mean square for all lookahead times between 0 and 90 minutes for queue prediction errors of time $i$.

$$
\sigma(i, *)=\sqrt{\sum_{j=0}^{90} e(i, j)^{2} / 90}
$$

Let $\sigma(*, j)$ be the root mean square for all queue prediction errors between time 120 and 240 at a lookahead time of $j$ minutes.

$$
\sigma(*, j)=\sqrt{\sum_{i=120}^{240} e(i, j)^{2} / 120}
$$

Let $\sigma(*, *)$ be the overall root mean square for all queue prediction errors between time 120 and 240 for all lookahead times between 0 and 90 minutes.

$$
\sigma(*, *)=\sqrt{\sum_{i=120, j=0}^{i=240, j=90} e(i, j)^{2} /(120 * 90)}
$$

Static departure rates assign a single departure rate for each runway that is used to set minimum separations for the strategic scheduler PTOTs used to calculate queue predictions. A range of static departure rates was tested in increments of 0.1 departures per quarter hour. For each runway, the departure rate that minimized $\sigma(*, *)$ was selected. The calibrated static departure rates were found to be 7.3 and 7.6 departures per quarter hour for runways $18 \mathrm{C}$ and $18 \mathrm{~L}$ respectively.

Variable departure rates use a unique departure rate per runway per quarter hour. Variable departure rates were modeled after the simulated number of departures per quarter hour (as seen in Fig. 7 for $18 \mathrm{C}$ and similarly for 18L) plus a constant offset value. A range of offset values was tested. For 
each runway, the offset value that minimized $\sigma(*, *)$ was selected. The calibrated offset values applied to the variable departure rates were found to be 1.7 and 1.3 departures per quarter hour for runways $18 \mathrm{C}$ and $18 \mathrm{~L}$ respectively. Because these variable rates were derived from simulated departure rates after the fact, they represent a zero uncertainty prediction, setting an upper limit of expectations for departure queue predictability using variable departure rates.

Fig. 8 shows $\sigma(*, *)$ for all three queue definitions for each runway using static and variable departure rate modes. The improvement in queue prediction of variable over static departure rate mode is substantial. Not only does the use of variable departure rates reduce the overall root mean square error for both runways, but the errors are more similar between runways. Note that there is very little difference in predictability between queue definitions. As Fig. 6 shows, q1 is always larger than the other queue definitions. Therefore, one could argue that the predictability normalized by average queue length is actually best for q1. Either way, the remaining comparisons focus on $\mathrm{q} 1$ as the results are very similar between queue definitions.

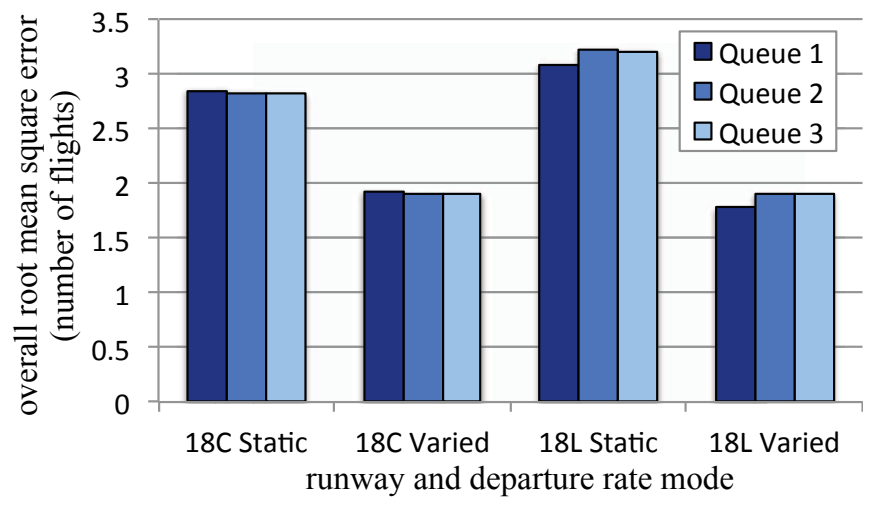

Fig. 8. Queue prediction overall root mean square error for the Baseline simulation.

Figs. 9 and 10 show $\sigma(*, j)$ and mean error, respectively, as a function of lookahead time for each runway and departure rate mode.

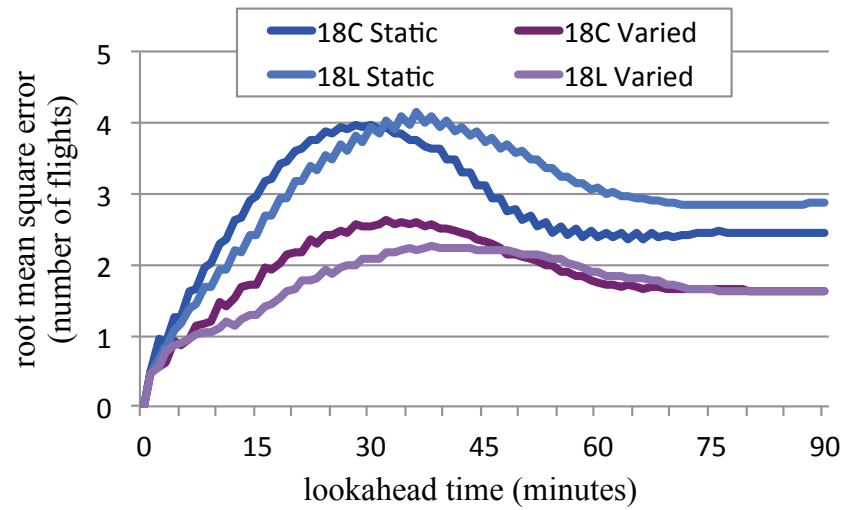

Fig. 9. Queue prediction root mean square error vs. lookahead time for the baseline simulation.
It is interesting to note that in all cases, the prediction root mean square error peaks around 30 minutes of lookahead time and then reduces, rather than continuing to increase with lookahead. The mean error also undulates with lookahead time and the undulations differ between runways. These root mean square and mean error effects are likely due to the dramatic peaks and valleys of the traffic demand. The use of variable departure rate mitigates these effects but does not eliminate them completely.

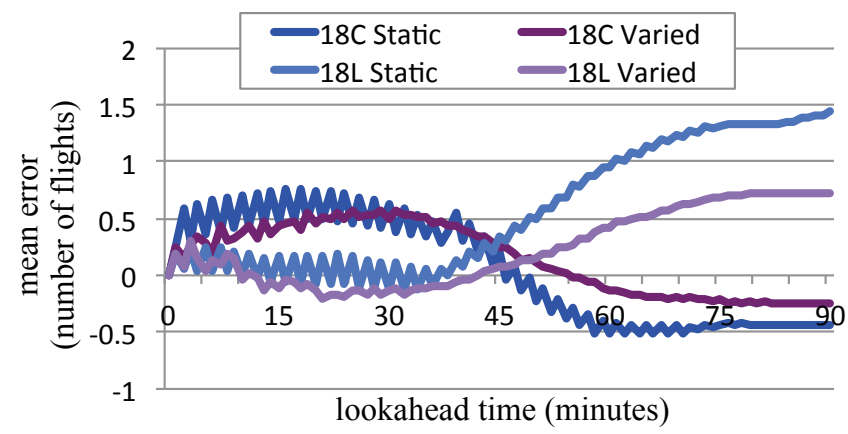

Fig. 10. Queue prediction mean error vs. lookahead time for the baseline simulation.

Fig. 11 and 12 show static and variable departure rate $\sigma(i, *)$, mean error and actual queue length as a function of prediction time for runways $18 \mathrm{C}$ and $18 \mathrm{~L}$ respectively. For each runway, the $\sigma\left(i,{ }^{*}\right)$ trends for static and variable departure rates are similar, although the variable departure rate errors are lower. In both cases, the $\sigma(i, *)$ values appear to be high and mean error low (under predicted queue length) when the actual queue length is high.

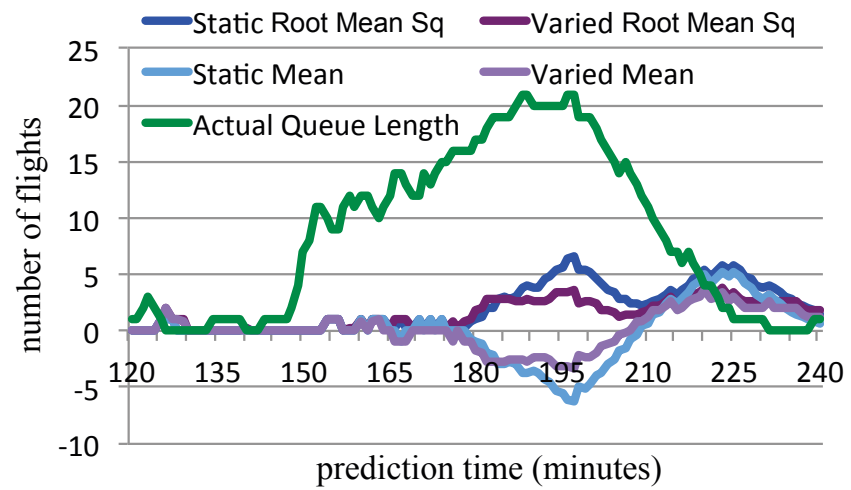

Fig. 11. Queue prediction root mean square and mean error vs. prediction time for runway $18 \mathrm{C}$ for the baseline simulation. 


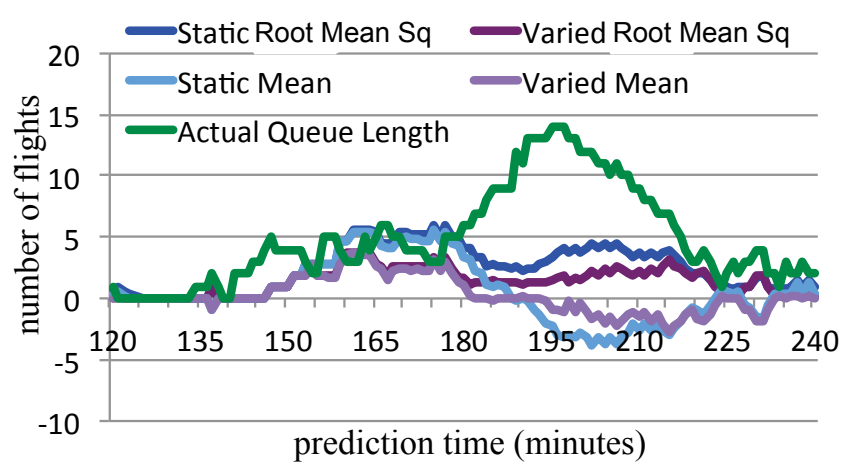

Fig. 12. Queue prediction root mean square and mean error vs. prediction time for runway $18 \mathrm{~L}$ for the baseline simulation.

It is clear that variable departure rate provides substantial improvement to queue prediction over static departure rates. However, the variable departures rates in this analysis were modeled after actual departure rates with no uncertainty. It is unclear if manually updated departure rates will enable the strategic scheduler to achieve this level of queue prediction accuracy and stability. Future research should explore automated methods of departure rate prediction to feed queue prediction. Future research should also explore incorporating any arrival data available in the strategic time frame directly into the strategic scheduler rather than relying on solely departure rate to approximate arrival impact.

When a similar analysis of queue predictability is performed on the simulation with tactical scheduling, queue prediction overall root men square error is nominally doubled. Fig. 13 shows how when lookahead time exceeds 15 minutes, the mean error rises rapidly and continues to over predict queue length.

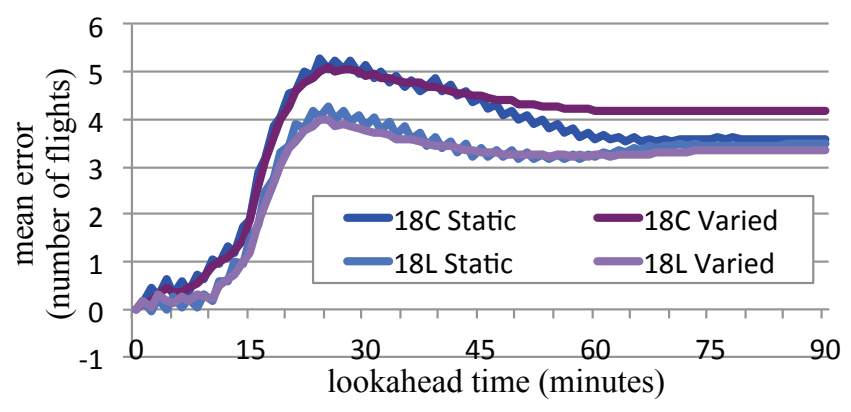

Fig. 13. Queue prediction mean error vs. lookahead time for the tactical scheduling simulation.

This is expected because the tactical scheduler actively meters the departures to lower queue lengths as seen in Fig. 6. Beyond 15 minutes lookahead, the strategic scheduler does not have the benefit of knowing what if any gate holds (TOBT) the tactical scheduler will assign to flights. This is why the next section analyzes TOBT predictability as a measure of strategic scheduler performance in the presence of tactical scheduling.

\section{TOBT Prediction Analysis}

In the simulation with tactical scheduling, flights push back from the gate according to the last TOBT assigned by the tactical scheduler such that AOBT is equal to this final tactical TOBT. Strategic TOBT error for a given flight can then be measured relative to AOBT. A flight's TOBT error can also change with time as strategic queue predictions and TOBT calculations are updated prior to AOBT. To facilitate an analysis of time varying TOBT error that is comparable between simulations with and without tactical scheduling, the flight's EOBT was chosen as a reference time for TOBT lookahead. Let $\varepsilon(f, j)$ be the TOBT error for flight $f$ calculated at a lookahead time of $j$ minutes prior to its EOBT.

$$
\varepsilon(f, j)=\operatorname{TOBT}(f, j)-\operatorname{AOBT}(f)
$$

As with the queue prediction analysis, root mean square error was chosen to measure TOBT predictability and calibrate target queue lengths that best align the strategic and tactical schedulers. Let $\rho(*, j)$ be the root mean square error for all flights with EOBT between 120 and 240 minutes calculated with a lookahead time of $j$ minutes prior to EOBT.

$$
\rho(*, j)=\sqrt{\sum_{\operatorname{EOBT}(f)=120}^{\operatorname{EOBT}(f)=240} \varepsilon(f, j)^{2} / N}
$$

where $N$ is the number of flights for which EOBT is between 120 and 240 minutes into the simulation.

Whereas overall root mean square error was used to calibrate static departure rates and variable departure rate offsets for queue prediction, the effects of tactical scheduling on TOBT prediction error with respect to lookahead time make overall root mean square error less suitable for calibrating target queue lengths for strategic TOBT calculation. Therefore, $\rho(*, 30)$ was used to calibrate target queue lengths, which will now be shown. To illustrate the target queue length calibration process, Figs. 14 and 15 show $\rho(*, j)$ and mean TOBT prediction error, respectively, as a function of lookahead time for a sample range of target queue lengths (0-6) for runway $18 \mathrm{C}$ using static departure rate and queue 1.

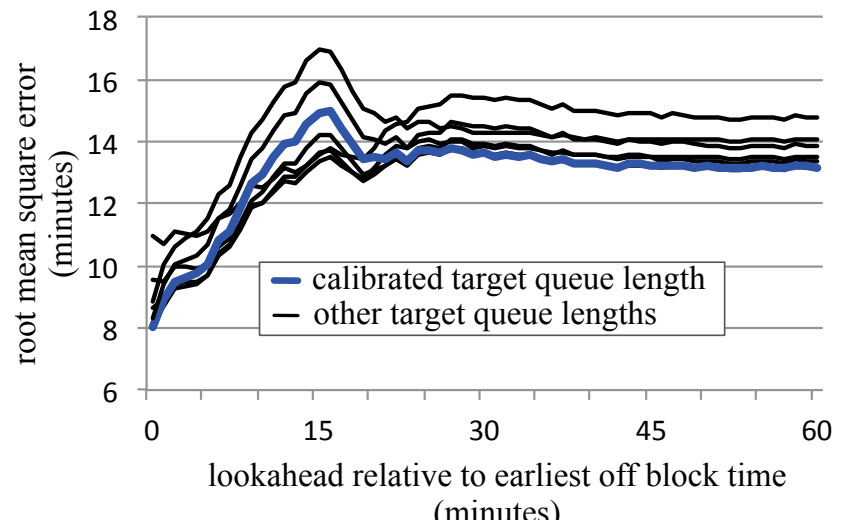

Fig. 14. Sample TOBT prediction root mean square errors vs. lookahead time for the tactical scheduling simulation. 


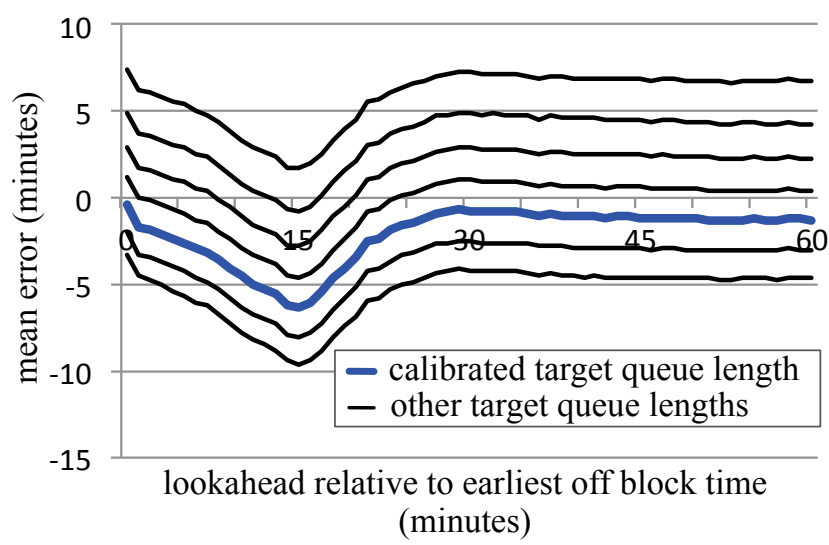

Fig. 15. Sample TOBT prediction mean errors vs. lookahead time for the tactical scheduling simulation.

Notice how both TOBT prediction error metrics show a distinct change in behavior around 15 minutes lookahead time, which is also the tactical scheduler planning horizon with respect to EOBT. At 15 minutes lookahead, the tactical scheduler assigns tactical TOBTs to flights, updating their EOBTs and trajectory predictions used for strategic queue prediction and TOBT calculation. Both strategic TOBT prediction error metrics stabilize around 30 minutes lookahead, which is why 30 minutes lookahead was chosen to calibrate target queue lengths. In Figs. 14 and 15 the target queue length with minimum root mean square error at 30 minutes lookahead is highlighted in blue.

Figs. 16, 17, and 18 show the calibrated target queue lengths and associated root mean square and mean error, respectively, for each queue definition and runway/departure rate mode.

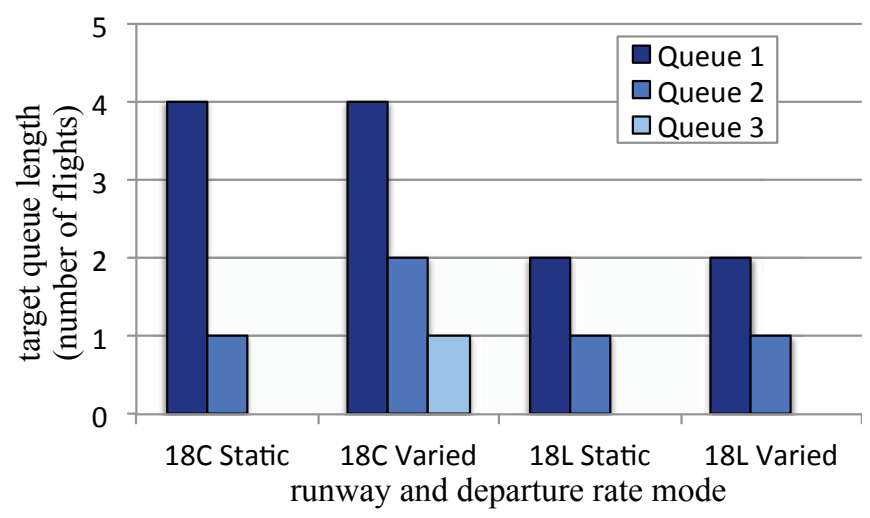

Fig. 16. Calibrated target queue lengths for the tactical scheduling simulation.

As expected, target queue lengths are greatest for q1 which counts the total number of flights active in ramp or taxi, and become smaller for $\mathrm{q} 2$ as the number of flights in ramp are removed, and smallest for $\mathrm{q} 3$ which focuses on the number of flights waiting in line for the runway. It is interesting to note that the calibrated target queue lengths are slightly smaller than average actual queue lengths produced by the tactical scheduler seen in Fig. 6. This may be because congestion related surface delays in the ramp and taxiways inflate the actual queues higher than the strategic scheduler predictions using unconstrained ramp and taxi transit times. In general the target queue lengths are small for the purposes of initiating and adjusting DMPs, which is the strategic schedulers intended function. For example, the DRM looks for queue predictions dipping below a user defined lower threshold to indicate the need for DMP compression. This would be impossible for the q3 target queue lengths of zero. In actual implementation, the strategic scheduler may be used more conservatively with higher target queue lengths to manage the level of residual delay the tactical scheduler will need to apply.

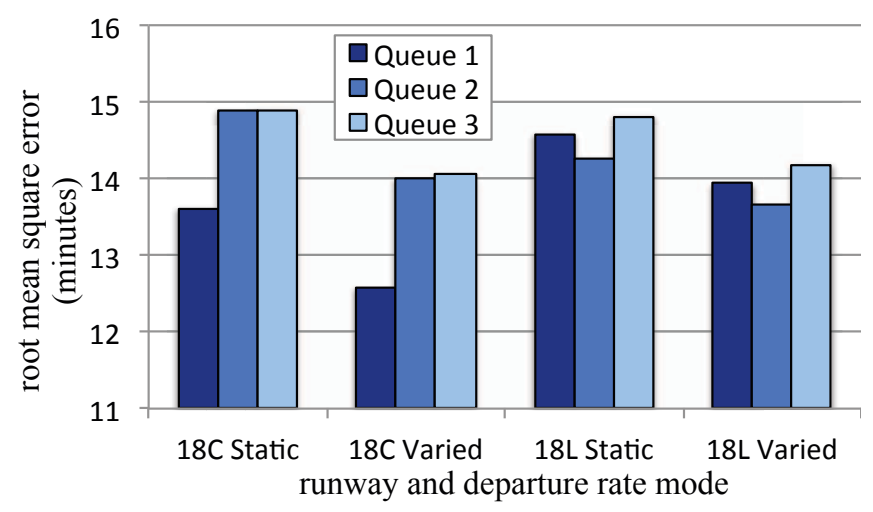

Fig. 17. TOBT prediction root mean square error at 30 minutes lookahead for calibrated target queue lengths for the tactical scheduling simulation.

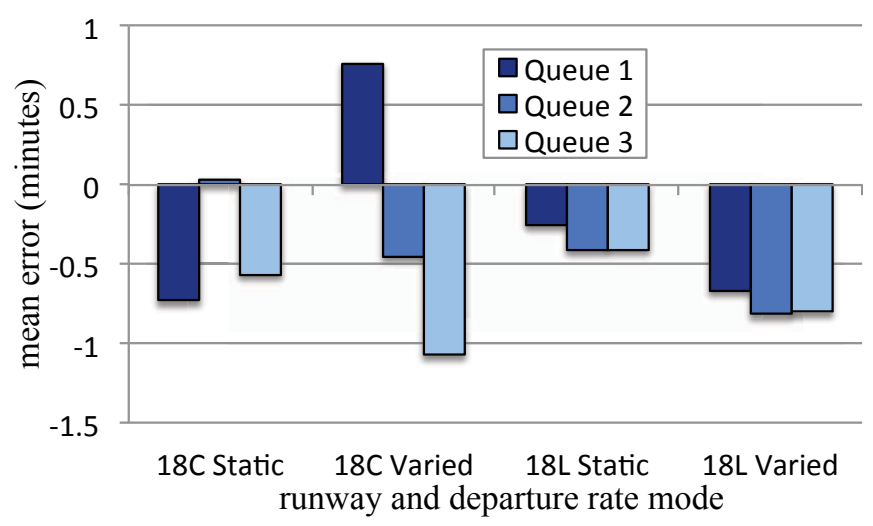

Fig. 18. TOBT prediction mean error at 30 minutes lookahead for calibrated target queue lengths for the tactical scheduling simulation.

Whereas mean TOBT prediction errors for most queue definitions are less than 1 minute, all root mean square errors are quite large (between 12 and 15 minutes). This is due to the tendency of the tactical scheduler to resequence flights at the runways in response to interactions with arrivals. This is especially true where gate conflicts between departure and arrivals are concerned. As the departure demand increases requiring more average gate delay, the likelihood of departures 
predicted to have a gate conflict with an arrival increases. This in turn increases the likelihood of early pushback to resolve gate conflicts changing the scheduled runway departure sequence. This tactical scheduler response to gate conflicts not only compounds tactical/strategic scheduler deviation, but leaves the system at risk to gaming if an airline should intentionally assign arrival gates to promote priority treatment of its departures. The tactical scheduler may be modified to plan for some delay to be absorbed in the ramp either by queuing at the spot or waiting in hard stands in response to gate conflicts rather than shifting to the delay to another flight and resequencing flights for runway usage.

\section{CONCLUSION}

This study analyzed strategic surface scheduler predictability to facilitate future integration with tactical scheduling. Queue prediction accuracy was used to measure strategic predictability in a simulation of CLT surface operations without tactical scheduling. The use of variable departure rates as a strategic scheduler input was shown to substantially improve queue predictions over static departure rates. This illustrates the importance of incorporating the anticipated effects of arrival operations on departures when scheduling without explicit arrival information.

A comparison of strategic assigned target gate push back times with actual gate pushback times was used to measure strategic predictability in a simulation with tactical scheduling. Whereas the strategic scheduler can be tuned to predict average delays similar to the tactical scheduler by calibrating target queue lengths, it is very difficult for the strategic scheduler to accurately predict individual flight delays assigned by tactical scheduler. This is due to the difference in flight sequence at the runway between the tactical and strategic schedulers. Reducing the effect of tactical departure resequencing in response to gate conflicts with arrivals is expected to reduce tactical/strategic scheduler deviation in future studies.

\section{ACKNOWLEDGMENT}

This research is funded by NASA's Airspace Operations and Safety Program in support of the Airspace Technology Demonstration 2 (ATD-2) subproject.

\section{REFERENCES}

[1] S. Zelinski and R. Coppenbarger, "A Concept for Integrated Arrival/Departure/Surface (IADS) Traffic Management for the Metroplex," Airspace Technology Demonstration 2 (ATD-2) ConOps Synopsis, NASA Ames Research Center, August 2015, unpublished.

[2] FAA Air Traffic Organization Surface Operations Office Directorate "U.S. Airport Surface Collaborative Decision Making (CDM) Concept of Operations (ConOps) in the Near-Term: Application of the Surface Concept at United States Airport," June 2014, unpublished.

[3] M. Hayashi, T. Hoang, Y. C. Jung, W. Malik, H. Lee, and V. L. Dulchinos, "Evaluation of Pushback Decision-Support Tool Concept for Charlotte Douglas International Airport Ramp Operations," Tenth USA/Europe Air Traffic Management R\&D Seminar, Lisbon, Portugal, June 2015.

[4] W. A. Malik and Y. C. Jung, "Exact and Heuristic Algorithms for Runway Scheduling," AIAA Aviation Confrence, Washington, D. C., June 2016.

[5] R. D. Windhorst, J. V. Montoya, Z. Zhu, S. Gridnev, K. Griffin, A. Saraf, and S. Stroiney, "Validation of Simulations of Airport Surface Traffic with the Surface Operations Simulator and Scheduler," $13^{\text {th }}$ Aviation Technology, Integration, and Operations (ATIO) Conference, Los Angeles, California, August 2013. 